\title{
Coronary calcification in adults with Turner syndrome
}

\author{
Marissa Schoepp, BS ${ }^{1}$, Fady Hannah-Shmouni, MD, FRCPC ${ }^{2}$, Jatin Matta, PA ${ }^{1}$, Ahmed M. Ghanem, PhD ${ }^{1}$, \\ John A. Hanover, $\mathrm{PhD}^{3}$, Khaled Z. Abd-Elmoniem, PhD, $\mathrm{MHS}^{1}$ and Ahmed M. Gharib, MD ${ }^{1}$
}

Purpose: Adults with Turner syndrome (TS) have an increased predisposition to ischemic heart disease. The quantitative relationship between coronary atherosclerosis and TS has yet to be established.

Methods: A total of 128 females (62 with TS) participated in this prospective study. Coronary computed tomography angiography was performed to measure coronary calcified plaque burden, and prevalent noncalcified plaque burden. Regression analysis was used to study the effects of TS and traditional cardiovascular disease risk factors on coronary plaque burden.

Results: Adults with TS were $63 \%$ more likely to have coronary calcifications than controls (odds ratio 1.63, 95\% confidence interval: $1.02,2.61, P=0.04$ ), with an age cutoff of 51.7 years for a probability of $>50 \%$ for the presence of coronary calcifications, when compared to 55.7 years in female controls. The average age of
TS patients with calcified plaques was significantly lower than that of controls with calcified plaques $(51.5 \pm 8.9$ years vs. $60.5 \pm 7.0$ years, $P<0.001)$. Age increased the likelihood of coronary calcifications by $13 \%$ per year (odds ratio 1.13 , confidence interval 95\%: 1.07-1.19, $P<0.001$ ).

Conclusion: This study demonstrates a higher prevalence and earlier onset of calcified coronary plaques in TS. These findings have important implications for cardiovascular risk assessment and the management of patients with TS.

GENETICS in MEDICINE advance online publication 2 October 2017

Key Words: atherosclerosis; coronary calcification; monosomy Turner syndrome

\section{INTRODUCTION}

Turner syndrome (TS) is one of the most common chromosomal disorders in females, characterized by partial or complete loss (monosomy) of one of the $\mathrm{X}$ chromosomes. This condition results in approximately 50 cases of TS per 100,000 female births. ${ }^{1,2}$ Patients manifest a broad phenotypic spectrum, including short stature, premature ovarian failure, and a variety of congenital anomalies. The most common congenital heart diseases include aortic coarctation (ACo) and bicuspid aortic valves (BAVs), ${ }^{3,4}$ In addition, patients with TS have an increased predisposition to hypertension, dyslipidemia, obesity, diabetes mellitus, and vasculopathy. ${ }^{3-6}$

Cardiovascular disease, including ischemic heart disease, is the leading cause of death in women. Females with premature ovarian insufficiency (estrogen deficiency) present an increased risk for cardiovascular disease, probably due to an earlier onset of vascular endothelial dysfunction. Additional causative mechanisms of ischemic heart disease in TS may include multivessel atherosclerosis, embolism from intracardiac thrombus, vascular endothelial dysfunction, and coronary artery involvement in aortic dissection. ${ }^{7}$ Vascular malformations, particularly aortic aneurysm (standardized mortality ratio $23.6,95 \%$ confidence interval (CI); $13.8-37$ ), account for the majority of cardiovascular related deaths $(\sim 41 \%)$ in TS. ${ }^{2}$ However, the mortality from coronary artery disease (CAD) is also increased in TS (standardized mortality ratio 3.47, confidence interval (CI) 2.06-5.48). ${ }^{1}$ This highlights the need for early identification and quantification of CAD in this population.

Evaluating the amount of atherosclerotic disease in the coronary arteries (CAD) helps predict cardiovascular morbidity and mortality in the general population. ${ }^{8-11}$ Atherosclerotic plaque burden can be reliably quantified in coronary computed tomography angiography (CTA), using several anatomic scores. These coronary plaque burden scores include; Agatston calcium score (CaS), segment involvement score (SIS), stenosis severity score (SSS), and segment volume score (SVS). ${ }^{8-11}$ While CaS measures only the amount of coronary calcification, the other measures (SIS, SSS, and SVS) include the noncalcified form of atherosclerotic plaques and account for their numbers, degree of stenosis, and volumes, respectively. Hence, such comprehensive CTA-based scores serve as noninvasive surrogates for overall coronary atherosclerotic burden, and are predictive for future adverse cardiac events. $^{8-11}$ Despite the implications of premature coronary atherosclerosis in TS, ${ }^{1,12}$ CTA use has been limited to the assessment of coronary anomalies for this population. ${ }^{13,14}$ No prior studies have investigated the magnitude of CTA

${ }^{1}$ Biomedical and Metabolic Imaging Branch, National Institute of Diabetes and Digestive and Kidney Diseases, National Institutes of Health, Bethesda, Maryland, USA; ${ }^{2}$ Section on Endocrinology \& Genetics, National Institute of Child Health and Human Development, National Institutes of Health, Bethesda, Maryland, USA; ${ }^{3}$ Laboratory of Cell and Molecular Biology, National Institute of Diabetes and Digestive and Kidney Diseases, National Institutes of Health, Bethesda, Maryland, USA. Correspondence: Khaled Z. Abd-Elmoniem (abdelmoniemkz@mail.nih.gov)

Submitted 26 April 2017; accepted 18 July 2017; advance online publication 2 October 2017. doi:10.1038/gim.2017.149 
coronary plaque burden scores in asymptomatic adults with TS. In this study, we demonstrate the extent of coronary atherosclerosis plaque burden and the average age of involvement in TS when compared to female controls.

\section{MATERIALS AND METHODS}

A total of 128 women participated in this study. TS $(n=62)$ were nonmosaic $45 \mathrm{X}$, and all subjects were adults, asymptomatic, and without known history of CAD. All TS subjects were participants in a prospective observational study (ClinicalTrials.gov identifier: NCT00006334). Control volunteers were recruited under protocol NCT01399385. All subjects gave written informed consent. Body mass index (BMI) and Framingham risk score (FrS) were calculated for each group. CTA images were acquired using multi-detector computed tomography (CT) as previously described. ${ }^{14-16}$ Briefly, CaS was obtained without contrast, ${ }^{10}$ then followed by CTA using intravascular contrast. Axial, multiplanar reformatted coronary images were obtained using a threedimensional-software tool (Virtual Place, AZE, Tokyo, Japan), which had been previously validated for assessment of the coronary arteries. ${ }^{14,15}$

In each of the 16 coronary segments, three plaque characteristics were recorded:

1. Presence of plaque (no $=0$, yes $=1$ )

2. Plaque luminal diameter stenosis severity (none: $0 \%$ luminal stenosis $=0$, mild: $1-49 \%$ luminal stenosis $=1$, moderate: $50-69 \%$ luminal stenosis $=2$, or severe: $>70 \%$ luminal stenosis $=3$ )

3. Plaque volume (none $=0$, small/trace $=1$, mild $=2$, moderate $=3$, large $=4)^{9,11}$

To quantify the complete plaque burden for each subject, three scores were calculated based on CTA data (SIS, SSS, and SVS). SIS was calculated as the total number of coronary artery segments exhibiting plaque $\quad($ minimum $=0$; maximum =16); SSS was the sum of all the stenosis scores of individual segments (minimum $=0$; maximum $=48$ ), a measure of the subject's overall plaque extent into coronary lumen; ${ }^{11}$ and SVS was the sum of plaque volume scores of all the individual segments (minimum $=0$; maximum $=64$ ), a measure of the subject's total plaque volume along the

Table 1 Descriptive data in the Turner syndrome and control groups

\begin{tabular}{|c|c|c|c|c|}
\hline Parameters & All subjects $(N=128)$ & Turner syndrome $(N=62(48.4 \%))$ & Controls $(N=66(51.6 \%))$ & $P$ value \\
\hline \multicolumn{5}{|c|}{ Physical examination and demographic data } \\
\hline Age (years) & $46.90 \pm 12.34$ & $47.33 \pm 9.23$ & $46.50 \pm 14.73$ & 0.873 \\
\hline Body mass index $\left(\mathrm{kg} / \mathrm{m}^{2}\right)$ & $27.82 \pm 7.34$ & $28.67 \pm 8.69$ & $27.01 \pm 5.75$ & 0.734 \\
\hline Systolic blood pressure $(\mathrm{mm} \mathrm{Hg})$ & $121.80 \pm 15.93$ & $120.77 \pm 17.68$ & $122.76 \pm 14.16$ & 0.238 \\
\hline Triglycerides (mg/dl) & $112.97 \pm 66.76$ & $116.23 \pm 73.69$ & $109.91 \pm 59.94$ & 0.610 \\
\hline LDL cholesterol (mg/dl) & $103.44 \pm 32.00$ & $95.56 \pm 31.24$ & $110.83 \pm 31.15$ & 0.522 \\
\hline HDL cholesterol (mg/dl) & $59.63 \pm 17.46$ & $60.92 \pm 20.36$ & $58.41 \pm 14.26$ & 0.865 \\
\hline Total cholesterol (mg/dl) & $186.96 \pm 34.74$ & $183.61 \pm 34.87$ & $190.11 \pm 34.60$ & 0.905 \\
\hline Framingham risk score & $1.36 \pm 1.79$ & $1.05 \pm 1.35$ & $1.65 \pm 2.10$ & 0.952 \\
\hline \multicolumn{5}{|l|}{ Race, N (\%) } \\
\hline Caucasian & $88(68.8 \%)$ & $52(83.9 \%)$ & $36(54.5 \%)$ & 0.002 \\
\hline African-American & $26(20.3 \%)$ & $6(9.7 \%)$ & $20(30.3 \%)$ & \\
\hline Other & $14(10.9 \%)$ & $4(6.5 \%)$ & $10(15.2 \%)$ & \\
\hline \multicolumn{5}{|l|}{ Medical history, N (\%) } \\
\hline Hypertension & $52(40.6 \%)$ & $30(48.4 \%)$ & $22(33.3 \%)$ & 0.106 \\
\hline History of smoking & $23(18.0 \%)$ & $6(9.7 \%)$ & $17(25.8 \%)$ & 0.022 \\
\hline Dyslipidemia $^{a}$ & $56(43.8 \%)$ & $24(38.7 \%)$ & $32(48.5 \%)$ & 0.289 \\
\hline Bicuspid aortic valve & $15(11.7 \%)$ & $15(24.2 \%)$ & $0(0.0 \%)$ & $<0.001$ \\
\hline Coarctation of aorta & $7(5.5 \%)$ & $7(11.3 \%)$ & $0(0.0 \%)$ & $<0.001$ \\
\hline \multicolumn{5}{|l|}{ Coronary imaging measurements ${ }^{b}$} \\
\hline Calcified plaque, $N(\%)$ & $42(32.8 \%)$ & $24(38.7 \%)$ & $18(27.3 \%)$ & 0.191 \\
\hline Calcium score (Agatston units) ${ }^{c}$ & $0(0-11.75)$ & $0(0-32.75)$ & $0(0-0.875)$ & 0.502 \\
\hline SIS* & $4.5(2.2-7)$ & $4(1.8-7)$ & $5(3-8)$ & 0.960 \\
\hline $\mathrm{SIS}>5, N(\%)$ & $44(34.4 \%)$ & $19(30.6 \%)$ & $25(37.9 \%)$ & 0.458 \\
\hline $\mathrm{SSS}^{*}$ & $7(2-12)$ & $7.5(2-14)$ & $6(2-12)$ & 0.539 \\
\hline SSS $>5, N(\%)$ & $71(55.5 \%)$ & 37 (59.7\%) & $34(51.5 \%)$ & 0.378 \\
\hline SVS* & $5(3-8)$ & $5(1.8-7.2)$ & $5(3-9)$ & 0.912 \\
\hline
\end{tabular}

HDL, high-density lipoprotein; LDL, low-density lipoprotein; SIS, segment involvement score; SSS, segment stenosis score; SVS, segment volume score.

a Serum lipids were measured in a morning fasting blood sample. Dyslipidemia was defined as any of the following abnormalities: total cholesterol $>200 \mathrm{mg} / \mathrm{dl}$, LDL $>160 \mathrm{mg} / \mathrm{dl}, \mathrm{HDL}<40 \mathrm{mg} / \mathrm{dl}$, or triglycerides $>150 \mathrm{mg} / \mathrm{dl}$. b ${ }^{b}$ ata are represented as means \pm standard deviations, except for ${ }^{\mathrm{C}}$ which are represented as medians, with interquartile ranges in parentheses. 

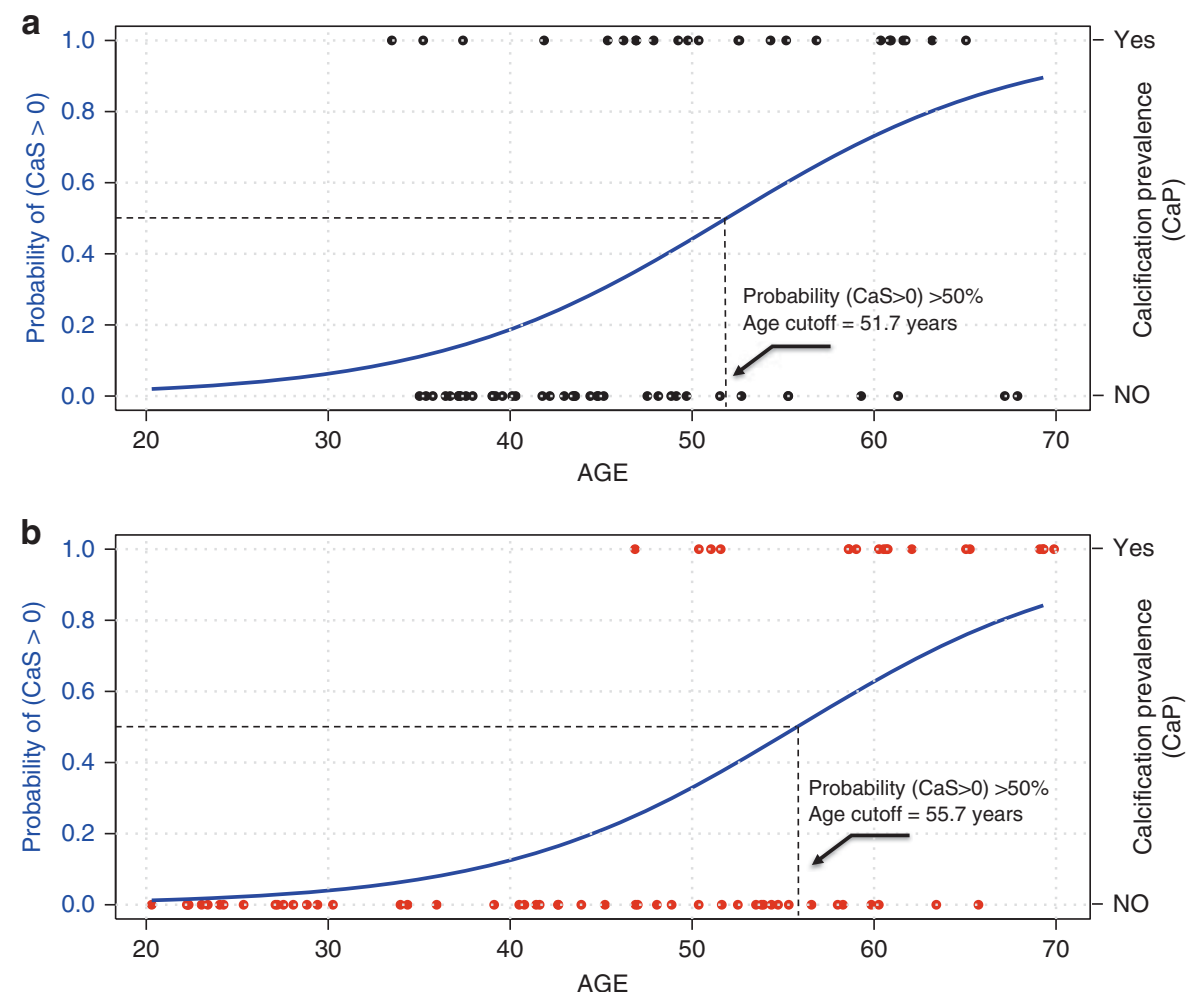

C

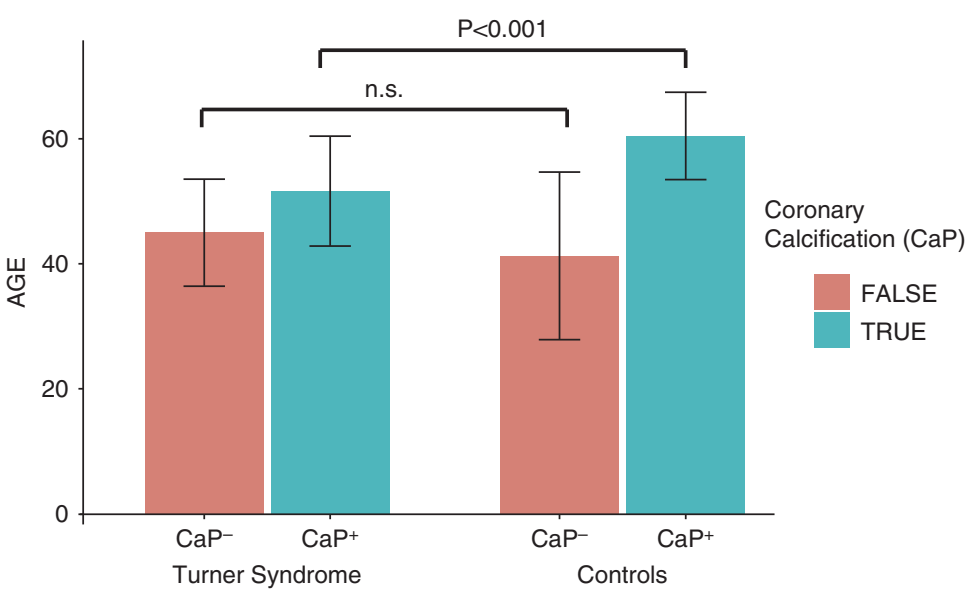

Figure 1 Predictive probability curves and bar graph. Predictive probability curves after adjusting for age and Framingham Risk Score. The probability of $>50 \%$ coronary calcifications were at age 51.7 years for patients with Turner syndrome when compared with 55.7 years for female controls (a and $\mathbf{b}$ ). Bar graph showing the average age of patients with Turner syndrome who had calcified plaques was significantly lower than that of controls with calcified plaques (c).

coronary vasculature. ${ }^{9,11}$ In addition to the continuous quantification of calcium $(\mathrm{CaS}),{ }^{10}$ the presence or absence of calcified plaques $(\mathrm{CaP})$ was further quantified $($ no $=0$, yes $=1)$. The presence of BAV, ACo, and ascending aortic dilation (defined as body surface area-corrected aortic diameter; aortic size index $(\mathrm{AI})>2 \mathrm{~cm} / \mathrm{m}^{2}$; aortic diameter $(\mathrm{cm})$ divided by body surface area $\left(\mathrm{m}^{2}\right)=$ ASI $\left.\left(\mathrm{cm} / \mathrm{m}^{2}\right)\right)^{17}$ were recorded for patients with TS.

We used a generalized linear model with binomial distribution and logit link function to evaluate the associations between atherosclerotic plaque scores (SIS, SSS, and
SVS), CaS, CaP, and cardiovascular risk factors. Univariable and multivariable generalized linear regression analyses were used to determine the association of TS status, cardiovascular risk factors, and each coronary plaque burden score. Final models of the continuous scores (SIS, SSS, and SVS) were represented by predictors' coefficient values and standard error. Final models of the binary scores $(\mathrm{CaS}, \mathrm{CaP}$, SIS $>5$, and SSS $>5$ ) were represented by the predictors' coefficients (standard error), odds ratio, and CI. $P \leq 0.05$ was considered statistically significant. A subset of TS with BAV, ACo, and dilated aorta (AI $>2 \mathrm{~cm} / \mathrm{m}^{2}$ ) was compared with TS 
without these conditions using analysis of covariance and Fisher tests.

\section{RESULTS}

Table 1 summarizes the baseline demographic, laboratory, and imaging characteristics of the entire cohort. Age, BMI, hypertension, and FrS were similar in the two groups. The control group had a higher percentage of African-American subjects and participants with a history of smoking. For the entire cohort, age was the only common independent predictor for plaque burden and calcification in the final multivariable regression analysis. Moreover, AfricanAmerican ethnicity was associated with a significantly increased SSS $(P<0.05)$ and SVS $(P=0.008)$ for plaque burden scores.

Participants with TS were more likely to have coronary calcifications than controls (odds ratio 1.63, 95\% CI: 1.02, 2.61, $P=0.04$; Figure 1a). After adjustment for age and FrS, the probability of $>50 \%$ coronary calcification was at age 51.7 years for TS, compared with 55.7 years for female controls (Figure 1a,b). The average age of TS with calcified plaques was significantly lower than of controls $(51.5 \pm 9.0$ years vs. $60.5 \pm 7.0$ years, $P<0.001$; Figure 1c). Age was the strongest variable associated with severe plaque burden using SIS $>5$ in TS (odds ratio 1.07, 95\% CI: 1.03-1.11, $P<0.001$ ). Aortic dilation was present in $39 \%(24 / 62)$ of patients with TS. Compared to TS without aortic dilation, after adjusting for age, this subset of patients demonstrated a higher prevalence of coronary calcifications (67\% vs. $21 \%$ ) and CaS (median 48.9 vs. 0$)(P<0.05)$. A sensitivity analysis, including FrS to the list of potential predictors, showed similar results (data not shown).

\section{DISCUSSION}

This study demonstrates a significant age-dependent difference in coronary atherosclerosis in TS, using several CTAbased plaque burden scores. These scores serve as validated surrogates for overall atherosclerotic burden in coronary arteries and are predictive for future adverse cardiac events. $^{8-11}$ This study shows that when matched for age, BMI, and FrS, adults with TS have a greater burden of coronary atherosclerosis and are younger compared to controls $(51.6 \pm 8.8$ years vs. $60.4 \pm 7.0$ years, $P<0.001)$. African-American subjects were underrepresented in the TS group, as is the case in many studies related to TS. However, race was included in the statistical models and was not an independent predictor for the presence of coronary calcium $(\mathrm{CaP})$. The association between $\mathrm{TS}$ and $\mathrm{CaP}$ was also independent of the other known cardiac risk factors. This is in keeping with the notion that adults with TS are at an increased risk for cardiovascular disease, which is also probably exacerbated by their increased predisposition to hypertension, obesity, diabetes mellitus, ${ }^{3}$ dyslipidemia, ${ }^{6}$ and vasculopathy. ${ }^{5}$ Thus, a careful cardiovascular risk assessment in these patients is required to prevent future adverse cardiovascular events.
Despite the increased awareness of increased mortality in TS from ischemic heart disease and CAD, $, 1,2,4,12$ a quantitative and qualitative (calcified versus noncalcified) examination of the coronary atherosclerotic burden in TS has not been fully elucidated. Chromosomal abnormalities, including monosomy X, have been implicated in the development of cardiac calcifications $^{18}$ and altered immune activation, ${ }^{19}$ thereby possibly affecting the natural progression of CAD. This might explain, in part, our observation of an earlier age at onset of coronary calcification in TS compared to controls in the absence of a significant difference in overall coronary plaque burden measures (i.e., SIS, SSS, SVS).

The development of CaP in adults with TS might be linked to other vascular abnormalities, such as BAV, ACo, or aortic dilation, as seen in TS. Indeed, this was verified in the subset analysis of patients with TS and aortic dilation (AI $>2 \mathrm{~cm} /$ $\mathrm{m}^{2}$ ), who demonstrated a significantly higher prevalence of $\mathrm{CaP}$ and $\mathrm{CaS}$ than in patients with TS and normal AI after adjusting for age. Individuals with TS have estrogen deficiency, which contributes to intimal thickening, decreased production of nitrogen oxide in the vasculature, and increased levels of plasma lipoproteins, which are atherogenic., Estrogen and progestogen therapy has been shown to reduce intimal thickening and increase high-density lipoprotein cholesterol levels, ${ }^{20}$ which raises the question whether elevations in high-density lipoproteins will help decrease cardiac events in TS, although this is mere conjecture at present. Thus, further studies are needed to assess the effects of hormone replacement therapy on the cardiovascular system in TS.

These results have beneficial implications and may guide clinical screening and counseling of adults with TS for cardiovascular disease. Our results indicate that patients with TS have a greater burden of coronary calcifications at an earlier age compared to controls, which argues for heightened cardiovascular assessment using CTA in this population, especially since the typical symptoms of CAD may not be present. Moreover, CTA measurements may be considered a valuable clinical parameter in addition to the recommended usual care for TS. Although CT is mentioned in these recommendations for aortic evaluation in TS, there tends to be a bias towards Magnetic Resonance and/or echocardiogram imaging as first line, primarily to avoid radiation exposure, with the drawback that they do not assess the coronary vasculature. ${ }^{4}$ In particular, patients with TS who exhibit aortic dilation $\left(\mathrm{AI}>2 \mathrm{~cm} / \mathrm{m}^{2}\right.$ ) on these modalities might benefit from CTA for coronary plaque burden measurements that may alter a patient's clinical course. Thus, these findings may modify cardiovascular risk assessment and management of patients with TS.

Our study has several limitations. First, phenotypic analysis of TS due to other genetic defects, such as mosaicism, deletions, or X chromosome parental origin, was not available for examination. Second, this pilot study was designed to characterize the burden of coronary atherosclerosis in patients with TS in comparison to controls, and in relation to 
traditional coronary artery atherosclerosis risk factors (such as age, FrS, and dyslipidemia). The current data set is not sufficient to perform a specific analysis unique to TS, such as measuring the duration of hypertension and dosage or duration of oral hormone replacement therapy, without compromising statistical integrity. Thus, further studies with a larger cohort of patients with TS are necessary to achieve a statistical power that will establish causality. Third, African Americans with TS were underrepresented and larger studies are required to address this ethnic gap. Our findings support the use of CT as a modality for cardiovascular risk evaluation of patients with TS, as endorsed for the general population by the 2013 ACC/AHA. ${ }^{8}$

In conclusion, this study demonstrates that adults with TS have a higher prevalence and earlier onset of calcified coronary atherosclerosis than controls. Additionally, TS patients with aortic dilation have a higher prevalence of coronary calcifications and calcium score than patients with TS and normal AI. The increased coronary calcifications might have predictive value in studying the cardiovascular risk assessment, managing, implementing early lifestyle changes, and counseling for TS. Further studies with larger TS numbers are needed to better understand the effects of specific factors related to this population (e.g., genotype and hormone replacement therapy) on CAD.

\section{ACKNOWLEDGMENTS}

This work was supported by the Intramural Research Programs of the National Institute of Diabetes and Digestive and Kidney Diseases (NIDDK) and the Eunice Kennedy Shriver National Institute of Child Health and Human Development (NICHD). We honor the memory of Carolyn Bondy, who supported this research effort and dedicated her professional career to caring for patients with TS.

\section{DISCLOSURE}

The authors declare no conflict of interest.

\section{REFERENCES}

1. Stochholm K, Juul S, Juel K, Naeraa RW, Gravholt CH. Prevalence, incidence, diagnostic delay, and mortality in Turner syndrome. J Clin Endocrinol Metab 2006;91:3897-3902.

2. Schoemaker MJ, Swerdlow AJ, Higgins CD, Wright AF, Jacobs PA, Mortality in women with Turner syndrome in Great Britain: a national cohort study. J Clin Endocrinol Metab. 2008;93:4735-4742.

3. Sybert VP, McCauley E. Turner's Syndrome. N Engl J Med. 2004;351: 1227-1238.
4. Bondy CA. Care of girls and women with Turner syndrome: a guideline of the Turner Syndrome Study Group. J Clin Endocrinol Metab 2007;92: $10-25$.

5. Ostberg JE, Donald AE, Halcox JP, Storry C, McCarthy C, Conway GS. Vasculopathy in Turner syndrome: arterial dilatation and intimal thickening without endothelial dysfunction. J Clin Endocrinol Metab. 2005;90:5161-5166.

6. Van PL, Bakalov VK, Bondy CA. Monosomy for the X-chromosome is associated with an atherogenic lipid profile. J Clin Endocrinol Metab. 2006:91:2867-2870.

7. Mortensen $\mathrm{KH}$, Andersen NH, Gravholt CH. Cardiovascular phenotype in Turner syndrome-integrating cardiology, genetics, and endocrinology. Endocr Rev. 2012;33:677-714.

8. Goff DC Jr., Lloyd-Jones DM, Bennett G, et al. 2013 ACC/AHA Guideline on the assessment of cardiovascular risk: a report of the American College of Cardiology/American Heart Association Task Force on Practice Guidelines. Circulation. 2014;129(25 Suppl 2):S49-S73.

9. Hadamitzky M, Achenbach S, Al-Mallah M, et al. Optimized prognostic score for coronary computed tomographic angiography: results from the CONFIRM registry (COronary CT Angiography EvaluatioN For Clinical Outcomes: An InteRnational Multicenter Registry). J Am Coll Cardiol 2013;62:468-476.

10. Agatston AS, Janowitz WR, Hildner FJ, Zusmer NR, Viamonte M Jr., Detrano R. Quantification of coronary artery calcium using ultrafast computed tomography. J Am Coll Cardiol 1990;15:827-832.

11. Min JK, Dunning A, Lin FY, et al. Age- and sex-related differences in allcause mortality risk based on coronary computed tomography angiography findings results from the International Multicenter CONFIRM (Coronary CT Angiography Evaluation for Clinical Outcomes: An International Multicenter Registry) of 23,854 patients without known coronary artery disease. J Am Coll Cardiol 2011;58:849-860.

12. Bondy CA. Aortic coarctation and coronary artery disease: the $X Y$ factor. Circulation. 2012;126:5-7.

13. Viuff $\mathrm{MH}$, Trolle $\mathrm{C}$, Wen J, et al. Coronary artery anomalies in Turner Syndrome. J Cardiovasc Comput Tomogr. 2016;10:480-484.

14. Polkampally PR, Matta JR, McAreavey D, Bakalov V, Bondy CA, Gharib AM. Aneurysmal dilatation of medium caliber arteries in Turner syndrome. Congenit Heart Dis. 2011;6:382-383.

15. Neary NM, Booker OJ, Abel BS, et al. Hypercortisolism is associated with increased coronary arterial atherosclerosis: analysis of noninvasive coronary angiography using multidetector computerized tomography. J Clin Endocrinol Metab. 2013;98:2045-2052.

16. Gharib AM, Pettigrew RI, Elagha A, et al. Coronary abnormalities in hyper-IgE recurrent infection syndrome: depiction at coronary MDCT angiography. AJR Am J Roentgenol. 2009;193:W478-481.

17. Matura LA, Ho VB, Rosing DR, Bondy CA. Aortic dilatation and dissection in Turner syndrome. Circulation 2007;116:1663-1670.

18. Sahlin E, Sirotkina M, Marnerides A, Iwarsson E, Papadogiannakis N. Fetal calcifications are associated with chromosomal abnormalities. PLoS One. 2015;10:e0123343

19. Abramowitz LK, Olivier-Van Stichelen S, Hanover JA. Chromosome imbalance as a driver of sex disparity in disease. J Genomics. 2014;2: 77-88.

20. Ostberg JE, Storry C, Donald AE, Attar MJH, Halcox JPJ, Conway GS. A dose-response study of hormone replacement in young hypogonadal women: effects on intima media thickness and metabolism. Clin Endocrinol 2007;66:557-564. 